\title{
LA AUTOESTIMA Y LOS VALORES ORGANIZACIONALES EN ESTUDIANTES UNIVERSITARIOS Y NO ESTUDIANTES UNIVERSITARIOS DE LIMA
}

\author{
Dr. Alejandro E. Loli Pineda \\ Dra. Ernestina López Vega
}

La autoestima y los valores organizacionales en estudiantes universitarios y no estudiantes universitarios de Lima, es un estudio desarrollado en una muestra de 3215 estudiantes pertenecientes a cuatro universidades de Lima; y no estudiantes conformados por egresados, profesionales y postgraduado s, muchos de los cuales ostentan cargos directivos en entidades públicas o privadas, con el objeto de conocer la relación existente entre la autoestima y los valores organizacionales de los mismos, en Lima Metropolitana, y determinar las diferencias entre la autoestima y ciertos rasgos demográficos. Los resultados indican que los estudiantes universitarios le dan mayor importancia a los valores como servicio al cliente, la limpieza, y a la calidad del producto y del servicio, y menor importancia a la honradez, al respeto a la persona y a la responsabilidad; mientras que los no estudiantes le otorgan mayor importancia al servicio al cliente, limpieza y confianza, y menor importancia a honradez, puntualidad y responsabilidad. Esta misma jerarquía se observa cuando se establece la relación con la autoestima, sobre todo, cuando la auto estima es muy baja, baja o intermedia; mientras que cuando la autoestima es alta o muy alta se ubica en primer lugar la limpieza y mejora la puntualidad hasta ubicarse en el cuarto lugar en importancia, éste es un valor que mejora a medida que la autoestima se eleva.

Igualmente, se encuentran diferencias significativas cuando se compara la autoestima y algunos rasgos demográficos tales como la procedencia, el género, el estado civil como la edad.

\section{PALABRAS CLAVES:}

Autoestima, valores organizacionales, estudiantes universitarios y no estudiantes universitarios

The selfesteem and the organizationals values in university students and not university students of Lima, is a study developed in a sample of 32 I 5 students belonging to four universities of Lima; and not students conformed by egressions, professional and postgraduados, many of which show off directive charges in public or private companies, with the purpose to know the existing relation among the selfesteem and the values organizational of the same, in Lima Metropolitan, and to determine the differences among the autoestima and certain demographics characteristics. The results indicate that the university students give greater importance to the values as service to the client, the cleaning, and to the quality of the product and of the service, and smaller importance to the honesty, to the respect to the person and to the responsibility; while the not students offer greater importance to the service to the client, cleaning and confidence, and smaller importance to honesty,. This same hierarchy is observed when is established the relation with the selfesteem, above all, when the selfesteem is very drop, lowers or intermediate; while when the selfesteem is high or very high is located in the first place the cleaning and improvement the puntualidad to being located in the fourth place in importance, this is a value that improves to the extent that the selfesteem is elevated. Likewise, they are found differentiates significant when is compared the selfesteem and some demographics characteristics such as the origin, the kind, the civil state as the age.

KEY WORDS: Selfesteem, organisational values, university students and not university students. 


\section{INTRODUCCION}

Los estudios clásicos de la Psicología nos permiten determinar que tanto la autoestima como los valores tienen su base teórica en los primeros años de vida de las personas, base sobre las cuales se moldea la personalidad de acuerdo a las condiciones que ofrece el medio y a las posibilidades físicas, la inteligencia y el temperamento que posee la persona para admitir, limitar o rechazar las influencias de dicho ambiente (A. Loli y col., 1997, 1998). Del mismo modo, toda persona transmite sus conocimientos, ideas, fantasías, su manera de ser y actuar, influyendo en otras personas, grupos y organizaciones entre los que se mueve; de manera que las organizaciones no son sino el reflejo de estas interacciones e influencias mutuas. Como refiere Sherif y shenf (1969), la autoestima es el resultado que tiene el individuo de la "internalización de las normas y los valores del grupo social y de su adecuado desempeño".

Sin embargo, en los tiempos actuales, la globalización de la economía, la cultura y los avances tecnológicos en el mundo encontraron al Perú desposeída de conocimientos y recursos para enfrentarse a un nuevo orden de cosas. Las organizaciones que tienen la misión de producir bienes o servicios en bien de la sociedad saben que es impostergable el desarrollo integral del individuo, para hacer de él un sujeto competitivo, no solamente con la mejoría de sus habilidades para una electiva manipulación de la tecnología, sino, fundamentalmente, de su personalidad; es decir, impulsar el cambio de valores, creencias, hábitos y costumbres del pasado hacia actitudes y comportamiento de desarrollo, de calidad, si se quiere lograr productos y servicios de calidad.

Pero precisamente, en las últimas décadas, el Perú se vio envuelto en una crisis moral, política, económica y social más grave de su historia, comprometiendo valores fundamentales para una coexistencia sana en una sociedad mientras el mundo avanzaba a pasos acelerados hacia la globalización exigiendo de las organizaciones competitividad con calidad.

Investigaciones desarrolladas en otros países del mundo nos informan de la importancia que tienen los valores personales y organizacionales en el crecimiento de las instituciones. Peters y Waterman (1982); Curvin (1989); Cruz (1996) Y Rodríguez (1993), entre otros, habían demostrado que los recursos personales que el hombre tiene (habilidades y experiencia) son de vital importancia para el aprovecha-miento máximo de la tecnología, pero era aún más importante la personalidad de los miembros que laboran en las organizaciones, esencialmente la autoestima y los valores. Peters y. Waterman (1982) habían demostrado en un estudio de 43 empresas más exitosas de Estados Unidos de Norteamérica, que la clave de su éxito son ocho factores, de los cuales seis estaban vinculados a la naturaleza humana y todos ellos involucraban valores.

Hay quienes creen que es posible una gestión de calidad a través de los valores (Blanchard y O'Connor, 1997), y otros, tras reconocer que la cultura de una organización es vital para el éxito de las empresas identifican la cultura visible y la invisible; en la primera estarían comprendidos los estilos y los patrones de conducta de los empleados y, en la segunda, estarían los valores compartidos y los supuestos que se han sostenido a lo largo del tiempo, siendo éste lo más difícil de cambiar, mismos que tienen un gran impacto en el desempeño de las organizaciones (Korrer y Heskett, en James Stoner y otros, 1996). 
Por otro lado, Morante (1989) estudió las actitudes hacia el rol del trabajo y del hogar en madres trabajadoras y no trabajadoras y su relación con la autoestima, encontrando que un porcentaje ligeramente mayor de madres trabajadoras presentaban puntajes de nivel bajo, y las madres no trabajadoras, presentaban un nivel de autoestima aún más bajo, dándonos pie a creer que la autoestima estaba mejor conservada en madres trabajadoras por razones de trabajo. Greengless (1985), en una investigación sobre trabajo remunerado de la mujer y su relación con la autoestima, concluyó que el hecho que una mujer pueda contar con una remuneración, le sirve como un símbolo tangible de competencia, así encontró que las mujeres trabajadoras tenían mayores niveles de autoestima, auto confianza y mayor sentido de competencia personal y autonomía.

Peiró (1993) encuentra que el conflicto de función tiene relación negativa con la satisfacción laboral, el desempeño, el compromiso organizacional y la participación que da autoestima. Es decir, cuanto mayor es el conflicto de función menor es la satisfacción laboral, el compromiso organizacional y la participación que da autoestima.

La autoestima reforzada es capaz de elevar la productividad y calidad de un trabajador e ir mucho más allá para mejorar su actitud en el servicio elevando además su autoestima y el cambio personal hasta sentirse orgulloso, satisfecho con su empleo. "Este cambio de percepción laboral sólo es posible como establece Yerkes (1978) con el desarrollo de la autoestima hasta el nivel que Maslow (1952) señala como nivel de autorrealización personal, que nosotros llamamos nivel de servicio" (Cruz, 1997).

Cruz Ramírez (1997), plantea además que "el papel de los valores es crucial en todos los procesos de autoestima. Los valores son fuente de vida, inmutables y universales... Cuando los valores guían la autoestima, se alcanza el equilibrio ético ... La mayoría de los autores definen a la autoestima como una percepción de seguridad y confianza, ... con apoyo en los valores se nutre a la autoestima de un ser universal. Los valores unifican, unen a la gente en fines y metas comunes, respaldan el bien común en una relación ganar-ganar".

Sin embargo, la autoestima y los valores vistos de otro ángulo, diría que la autoestima sostiene a los valores, que es la base sobre la cual se construyen los valores (Loli y López, 1997), o, mientras la autoestima infunde energía, pasión, visión, etc. a la persona o al conjunto, los valores personales controlan y regulan su actuación (Mora, 1996).

Frente a estas disonancias, A. Loli, E. López, (1998), realizan una investigación sobre la relación entre la autoestima y los valores organizacionales en 105 gerentes de Microempresas ubicadas en el Cono Este de Lima Metropolitana, encontrando correlación entre autoestima alta y algunos valores, "cuanto más alta es la autoestima más importante es la lealtad pero menos importante es el amor al trabajo"; también se encontró correlación entre autoestima baja y otros valores, " ... cuanto más baja es la autoestima menos importante es la lealtad y el trabajo en equipo". Igualmente, se compararon los valores organizacionales separando la muestra por lugar de procedencia, género, grado de instrucción y actividades de la empresa, encontrando diferencias significativas en cada situación, demostrando la influencia de estas variables en la percepción de los valores organizacionales por los gerentes, que tienen que ver con su cultura, sus conocimientos y el tipo de actividad de sus empresas. 
Por otro lado, respecto a la jerarquización de los valores organizacionales por los gerentes, de los 36 valores puestos en prueba, los 10 más importantes son aquellos de más baja valoración; es decir, a pesar de ser más importantes dichos valores, son menos tomados en cuenta en la práctica organizacional (Loli y Col., 1998). Más tarde (1999) los mismos autores en una muestra de 1464 estudiantes universitarios de Lima encuentran correlación positiva entre la autoestima y el valor organizacional limpieza y correlación negativa entre la autoestima y los valores organizacionales responsabilidad y respeto a la persona. Estos resultados demuestran que existe relación entre la autoestima y los valores organizacionales en el proceso productivo (gerentes) y en la aspiración de los estudiantes de trabajar en una organización a la culminación de sus estudios.

Estos resultados llevaron a los autores a diseñar una nueva investigación. Se consideró que los resultados, si bien arrojaron la relación esperada entre autoestima y los valores organizacionales, surgió el interés por saber los cambios que pueden operarse con una muestra más amplia; es decir, conocer la relación existente entre la autoestima y los valores organizacionales de los estudiantes universitarios y no estudiantes universitarios (egresados, profesionales y postgraduados) de Lima metropolitana.

\section{METODOLOGIA}

La metodología empleada es la de Ranking (medida de variabilidad) para determinar la jerarquía de valores organizacionales y las diferencias de éstos de acuerdo a los niveles de autoestima; el puntaje " $t$ " de Student para determinar las diferencias de la autoestima con las variables demográficas en grupos independientes y finalmente se utilizó el Análisis de Varianza de un factor complementada mediante el Test de Scheffe para establecer específicamente entre qué pares de grupos se observan las diferencias.

\section{MUESTRA}

La muestra estuvo conformada por 3,215 sujetos. De ellos, 2,785 fueron estudiantes universitarios de cuatro universidades públicas y privadas de Lima y 430 fueron no estudiantes universitarios integrados por egresados, profesionales y postgraduado s, la mayoría de los cuales en pleno ejercicio de alguna función directiva en entidades públicas o privadas.

\begin{tabular}{|c|c|c|}
\hline \multicolumn{3}{|c|}{ COMPOSICIÓN DE LA MUESTRA } \\
\hline $\begin{array}{c}\text { ESTUDIANTES } \\
\text { UNIVERSITARIOS }\end{array}$ & NO ESTUDIANTES & TOTAL \\
\hline 2785 & 430 & 3215 \\
\hline
\end{tabular}

La muestra fue seleccionada de manera intencional tomando en cuenta la paridad de las carreras profesionales dentro de las universidades públicas y privadas a fin de mantener homogeneidad de estudiante; y en el caso de los egresados, profesionales y postgraduados por su condición de estar en pleno ejercicio de la actividad pública o privada y no ser estudiante universitario o haber dejado de serio. 


\section{TÉCNICAS DE RECOLECCION DE DATOS}

La técnica empleada para la recolección de la información fue la de administración colectiva de las pruebas en salones de clase, en el caso de los estudiantes; y mediante administración individual en los centros laborales, en el caso de los egresados, profesionales y postgraduados y sólo en algunos casos -en éstos últimos- en forma colectiva.

\section{INSTRUMENTOS}

La información se recolectó mediante el Inventario de Autoestima (ALPEL), Forma-AD y la lista de valores organizacionales (ALPEL), Forma-AV de Loli Pineda y López Vega.

El Inventario de Autoestima (ALPEL), Forma-AD es una prueba de 41 ítemes, conformado por cuatro factores (Autoconocimiento, autorrealización, autorrespeto y autoconfianza), validado sobre la base una muestra de 3,215 sujetos. El análisis estadístico sobre la validez de constructo indica la presencia de un solo factor que permite explicar el $37.60 \%$ de la varianza total. Esto nos indica que estos elementos estarían conformando de forma ordenada el constructo de la autoestima en nuestro medio.

Finalmente, el análisis de consistencia de todos los Ítemes que conforman el ALPEL Forma-AD obtiene un coeficiente de con fiabilidad Kuder-Richardson 20, de 0.84, lo que nos indica que la prueba permite obtener puntajes con fiables.

La lista de valores organizacionales se determinó a partir de la aplicación de una lista de 36 valores relacionados a las organizaciones, a una muestra de 105 gerentes de Microempresas, solicitándoles que establecieran una jerarquía de valores en orden de importancia, de los cuales, II fueron incorporados como instrumento de trabajo de la presente investigación.

\section{RESULTADOS}

\section{JERARQUIZACION DE LOS VALORES ORGANIZACIONALES EN ESTUDIANTES UNIVERSITARIOS Y NO ESTUDIANTES UNIVERSITARIOS}

El Cuadro $\mathrm{N}^{\circ} 1$ nos indica el orden de los promedios en que han sido evaluados los II valores organizacionales. De acuerdo a los estudiantes universitarios el valor más importante es el "servicio al cliente" $(8,89)$, seguido por "limpieza" $(8,87)$ y "calidad del producto y del servicio" (7,66); y los menos importantes son la "honradez" $(5,09)$, "respeto a la persona" $(3,82)$ y, "responsabilidad" $(3,76)$ de un total de 11 valores, quedando los demás en los niveles intermedios.

Cuando se analiza los 11 valores organizacionales en la percepción de aquellos que no son estudiantes universitarios, encontramos que los valores más importantes son el "servicio al cliente" (819), la "limpieza" (7,72) y la "confianza" (7,53), y los menos importantes son la "honradez" (5,22), la "puntualidad" (4,63) Y la "responsabilidad" (3,59).

Sin embargo, cuando se analiza la muestra total, los valores más importantes son el "servicio al cliente" $(8,79)$, la "limpieza" $(8,72)$ y la "calidad del producto o servicio" 
(7,56), mientras que los menos importantes son la "honradez" $(5,11)$, el "respeto a la persona" (4.10) y la "responsabilidad" $(3,74)$.

Por consiguiente, los estudiantes universitarios prefieren sacrificar los valores de honradez, respeto a la persona y la responsabilidad por darle un servicio al cliente, mantener la limpieza y que el producto o servicio sea de calidad. Sin embargo los sujetos que no son estudiantes (egresados, profesionales y postgraduados), si bien mantienen como valioso el servicio al cliente y la limpieza, éstos van ligados a la confianza, pero también sacrifican la honradez, la puntualidad y la responsabilidad; es decir, que prefieren sacrificar la puntualidad antes que el respeto a la persona, esto tiene algo de positivo, probablemente se debe a las influencias de la experiencia cultural de las organizaciones a las que pertenecen o a la sociedad peruana misma.

\section{JERARQUIZACION DE LOS VALORES ORGANIZACIO ALES POR NIVELES DE AUTOESTIMA}

El cuadro $\mathrm{N}^{\circ} 2$ nos indica la jerarquización de los valores organizacionales por niveles de autoestima (muy baja, baja, intermedia, alta, y muy alta) de los sujetos. Las personas de autoestima muy baja le dan más importancia al "servicio al cliente" $(8,77)$, "limpieza" $(8,27)$ y "calidad del producto o servicio" $(7,43)$, y menos importancia a la "honradez" $(5,1$ 7), "respeto a la persona" $(4,56)$ y "responsabilidad" $(3,95)$. Esta misma postura se mantiene en aquellos que tienen la autoestima baja e intermedia, observándose el desplazamiento sólo de dos valores, el valor "respeto a la persona" $(3,95)$ que se ubica en el último lugar en aquellos que tienen la autoestima baja y el valor "puntualidad" $(6,75)$ que se eleva al cuarto lugar y se mantiene en aquellos que tienen la autoestima alta. Sin embargo, en los que tienen la autoestima alta y muy alta, el valor "limpieza" (9,14 Y 9,05 respectivamente) ocupan el primer lugar, permitiéndonos deducir que a medida que la autoestima se eleva el valor limpieza prevalece con más fuerza que los demás valores, probablemente porque una elevación demasiado grande de la autoestima linda con los intereses personales y el narcisismo.

\section{LA AUTOESTIMA DE LOS ESTUDIANTES UNIVERSITARIOS Y NO ESTUDIANTES UNIVERSITARIOS}

En el Cuadro $\mathrm{N}^{\circ}$ 3, el análisis de la comparación de la autoestima en general indica que existen diferencias significativas entre los estudiantes universitarios y no estudiantes universitarios; observándose que los estudiantes universitarios alcanzan en promedio $(33,34)$ puntuaciones más elevadas que los no estudiantes universitarios $(31,30)$.

En la comparación de cada uno de los Factores también se observan que en todos los casos existen diferencias significativas, pero se aprecia que los estudiantes universitarios sólo superan en puntaje a los no estudiantes en el Factor 1 (autoconocimiento), en tanto que en los Factores 2, 3 Y 4 (autorrealización, autorrespeto y autoconfianza), los no estudiantes obtienen puntajes superiores a los alcanzados por los estudiantes universitarios. Probablemente, porque los estudiantes refuerzan su autoestima en el conocimiento que tienen de sí mismo por estar en proceso de formación; mientras que los no estudiantes prefieren sostener su autoestima más en la realización personal, el respeto y la confianza en sus actos, que les permite despegar y crecer. 


\section{LA AUTO ESTIMA Y ALGUNOS RASGOS DEMOGRAFICOS}

\subsection{La autoestima y el lugar de procedencia}

En el Cuadro $\mathrm{N}^{\circ} 4$, el análisis de la comparación de la autoestima en general indica que existen diferencias significativas entre los que proceden de Lima y los de provincia; observándose que los que proceden de Lima alcanzan en promedio $(33,30)$ puntuaciones más elevadas que los de provincia $(32,18)$.

En la comparación de cada uno de los Factores que conforman la autoestima, también se observa que en todos los casos existen diferencias significativas, con excepción del Factor 4 (autoconfianza) donde no existe diferencia significativa, lo que nos indica que tanto los de Lima como los de Provincia obtienen puntajes equivalentes en este aspecto.

\subsection{La autoestima y el género}

En el Cuadro $\mathrm{N}^{\circ} 5$, el análisis de la comparación de la autoestima en general, demuestra que existen diferencias significativas entre hombres y mujeres; observándose que los hombres alcanzan en promedio $(32,78)$ puntuaciones más bajas que las mujeres $(33,37)$.

En la comparación de cada uno de los Factores también se observa que en todos los casos existen diferencias significativas, pero se nota que los hombres sólo superan a las mujeres en el Factor 3 (autorrespeto), mientras que en los Factores 1, 2 Y 4 (autoconocimiento, autorrealización y autoconfianza) las mujeres obtienen puntajes superiores a los alcanzados por los hombres. Probablemente, porque los hombres se sienten más capaces de atender sus propias necesidades, sentimientos y actos; en tanto que las mujeres son más flexibles a los cambios, son amantes de la verdad y la justicia, capaces de realización y crecimiento, pero también con fiables.

\subsection{La autoestima y el estado civil}

En el Cuadro $\mathrm{N}^{\circ}$ 6, encontramos la comparación de la auto estima en general por Estado Civil y vemos que existen diferencias significativas entre los solteros y otros (casado, viudo, conviviente), observándose que los solteros alcanzan en promedio $(33,21)$ puntuaciones más elevadas que los casados, viudos y convivientes $(31,61)$.

En la comparación de cada uno de los Factores también se observa que en todos los casos existen diferencias significativas, pero se aprecia que los solteros sólo superan en puntaje a los otros en el Factor 1 (autoconocimiento), los demás Factores como 2, 3 Y 4 (autorrealización, autorrespeto y autoconfianza) los casados, viudos y convivientes obtienen puntajes superiores a los alcanzados por los solteros. Probablemente, porque la mayoría de la muestra de solteros son jóvenes estudiantes que sustentan su autoestima en su juventud y relativa libertad para actuar y adaptarse con facilidad al cambio, que aún están en condiciones de orientar su vida hacia metas positivas y claras, mientras que los casados, viudos y convivientes ya tienen una vida más estable y sostienen su autoestima en la realización y crecimiento psicológico sin causar daño ni expresar hostilidad a los demás por la confianza y fe que tiene sobre sí mismo y los demás.

\subsection{La autoestima y la edad}

En el Cuadro $\mathrm{N}^{\circ} 7$, encontramos el análisis comparativo de la autoestima por edad. A través del Análisis de Varianza de un Factor. En el caso de la autoestima general, se observa que existen diferencias significativas entre los grupos de edad estudiados, motivo 
por el cual fue necesario real izar las comparaciones a través del Test de Scheffe, que permite establecer específicamente entre qué pares de grupos se observan las diferencias. Los hallazgos indican que el grupo con participantes de 41 a más años de edad alcanzan puntajes menores $((30,22)$ que los participantes del grupo de 18 a 20 años de edad $(33,34)$, de 21 a 30 años de edad $(33,14)$ Y de 31 a 40 años de edad $(32,36)$.

El análisis Factor por Factor nos indica que en el Factor 1 (autoconocimiento), los puntajes obtenidos disminuyen a medida que aumenta la edad, posiblemente por que le otorgan menos peso a este Factor en la medida que sus expectativas han sido cubiertas y han logrado cierta estabilidad. Mientras tanto, en los Factores 2, 3 y 4 (autorrealización, autorrespeto y autoconfianza) los puntajes logrados se incrementan a medida que aumenta la edad, situación coherente con el análisis del Factor 1, donde la autorrealización, el respeto y la confianza en sí y en los demás son más importantes para sostener su autoestima.

\section{CUADRON" I \\ JERARQUIZACION DE LOS VALORES ORGANIZACIONALES POR SU CONDICION DE ESTUDIANTES Y NO ESTUDIANTES Y MUESTRA TOTAL.}

\begin{tabular}{|c|c|c|c|c|c|c|c|}
\hline $\mathrm{N}^{*}$ & ESTLDIANTE $(\mathrm{N}=$ & 2,785 & & NO ESTLDIANTE (N & $Y=430)$ & MUESTRA TOTAL. (N) & $=3,215)$ \\
\hline ORDEN & VALORES & M & $\mathrm{DE}$ & VALORES & II DE. & VALORES & II DE \\
\hline 1 & Servicio al cliente & 8,89 & $2.98 \mathrm{~s}$ & Servicio al cliente & $8.193,34$ & Servicio al cliente & $8,793,04$ \\
\hline 2 & Limpica & 8.87 & 2,951 & Limpieza & 7.723 .49 & Limpicza & $8,72,3,06$ \\
\hline 3 & Calidad del prod. o verv. & 7,66 & $3,4,30$ & Confianza & $7,53,3,03$ & Calidad del prod. o serv. & $7.56 \quad 3.50$ \\
\hline 4 & Puntualidad & 6,90 & 3,231 & Deseo de superacián & $7,10 \quad 3,10$ & Disciplina & $6.78 \quad 3.11$ \\
\hline 5 & Disciplina & 6.75 & 3,09 । & Disciplina & $6,973,23$ & Confianza & $6,73,3,10$ \\
\hline 6 & Confianza & 6.60 & $3,09($ & Calidad del prod. o serv. & $6,90 \quad 3,82$ & Puntualidad & $6.59 \quad 3.33$ \\
\hline 7 & Amor al trabajo & 6,41 & 3,28 & Amor al trabajo & 6.763 .25 & Amor al trabajo & $6,46 \quad 3,28$ \\
\hline 8 & Deseo de superaciòn & 6,02 & $3,08 \mathrm{H}$ & Respeto a la persoma & $5,94,3,11$ & Deseo de superación & $6.16 \quad 3,10$ \\
\hline 9 & Hoaradez & 5.09 & 2,901 & Honradez & 5.222 .81 & Honradez. & 5.112 .89 \\
\hline 10 & Respeto a la persona & 3,82 & $3,05 \mathrm{P}$ & Puntualidad & $4,63 \quad 3,31$ & Respeto & $4,10 \quad 3,14$ \\
\hline 11 & Responsabilidad & 3,76 & $2,34 \mathrm{~K}$ & Responsahilidad & $3,59 \quad 2,58$ & Responsabilidad & $3,7+2,37$ \\
\hline
\end{tabular}


CUADRO N"2

JERARQUIZACION DE LOS VALORES ORGANIZACIONALES POR NIVELES DE AUTOESTIMA

\begin{tabular}{|c|c|c|c|c|c|c|c|c|c|c|c|c|c|}
\hline \multicolumn{14}{|c|}{ AUTOESTIMA } \\
\hline \multicolumn{3}{|c|}{ MUY BAAA $\left(\mathbf{N}^{n} 700\right)$} & \multicolumn{3}{|c|}{ BAJA (N" 684$)$} & \multicolumn{3}{|c|}{ INTER MEDIA (N* 837$)$} & \multicolumn{3}{|c|}{ ALTA (N"606) } & \multicolumn{2}{|c|}{ MUY ALTA $\left(\mathrm{N}^{*} 388\right)$} \\
\hline VALORES & $M$ & DS & VALORES & $\mathbf{M}$ & DS & VAL.ORES & $M$ & DS & VALORES & $\mathbf{M}$ & DS & VALORES & M DS \\
\hline $\begin{array}{l}\text { Servielo al } \\
\text { Cliente }\end{array}$ & 8.77 & 3,11 & $\begin{array}{l}\text { Servicio al } \\
\text { cliente }\end{array}$ & 8.73 & 2,99 & $\begin{array}{l}\text { Servicio al } \\
\text { Cliente }\end{array}$ & 8.82 & 3,02 & Limpiera & 9,14 & 2,83 & Limpiera & $9.052,90$ \\
\hline Lin & 8,27 & 3,24 & t.impieza & 8.60 & 3,08 & t.in & 8,73 & 3,05 & $\begin{array}{l}\text { Servicia al } \\
\text { cliente }\end{array}$ & 9,06 & 2,85 & $\begin{array}{l}\text { Servielo al } \\
\text { cliente }\end{array}$ & $8,45,3,29$ \\
\hline $\begin{array}{l}\text { Calidad del } \\
\text { prod. Servie } \\
\text { Diseiplina }\end{array}$ & 7,43 & 3,54 & $\begin{array}{l}\text { Calldad del } \\
\text { prod. Servic } \\
\text { Puntualidad }\end{array}$ & 7.46 & 3,36 & $\begin{array}{l}\text { Calidad del } \\
\text { pred. Servic } \\
\text { Puntualidad }\end{array}$ & & & $\begin{array}{l}\text { Callidad del } \\
\text { prod. Servic } \\
\text { Puntualidad }\end{array}$ & 7,81 & & $\begin{array}{l}\text { Calidad del } \\
\text { prod. servic } \\
\text { Disciplina }\end{array}$ & $7,27 \quad 3,74$ \\
\hline Confianza & 7,03 & 3,10 & Disci & 6,75 & 3,36 & Disciplina & 6.80 & 3,23 & Conf & 6,70 & 3,26 & Confianza & $6,81 \quad 3,03$ \\
\hline & 6.99 & 3.13 & & 6.72 & 3,12 & & 6,72 & 3. & & 6,62 & 3,10 & & $6,78 \quad 3,04$ \\
\hline $\begin{array}{l}\text { Amer al } \\
\text { trabaje }\end{array}$ & 6.22 & 3,29 & Conflanza & 6,65 & 3,1 & Conflanra & 6,62 & 3,06 & Disciplina & 6. & $3,1,2$ & Am & 6.70 .3 .21 \\
\hline $\begin{array}{l}\text { Desco de } \\
\text { superación } \\
\text { Puntualidad }\end{array}$ & 6,17 & 3,10 & $\begin{array}{l}\text { Ame } \\
\text { trab } \\
\text { Deve }\end{array}$ & 6. & 3 & $\begin{array}{l}\text { Ami } \\
\text { trah } \\
\text { Des }\end{array}$ & 6.56 & 3 , & $\begin{array}{l}\text { Amo } \\
\text { trab. } \\
\text { Dese }\end{array}$ & 6,48 & 3,19 & $\begin{array}{l}\text { Deseo de } \\
\text { Superación } \\
\text { Punfualidad }\end{array}$ & $6,593,01$ \\
\hline Henradez & 6.15 & 3.49 & $\begin{array}{l}\text { superación } \\
\text { Hosradez }\end{array}$ & 6,15 & 3,17 & $\begin{array}{l}\text { superación } \\
\text { Honradez }\end{array}$ & 6.10 & 3,09 & $\begin{array}{l}\text { Superación } \\
\text { Hearadez }\end{array}$ & 5,98 & 3,10 & Honradex & 6.523 .28 \\
\hline Respete & 5.17 & 2,95 & sa. & 5.28 & 2,94 & Respeto & 5,00 & 2,86 & Respeto & 4,99 & 2,78 & Respeto & $5,10 \quad 2,92$ \\
\hline & 4,56 & 3.18 & bilidad & 3.97 & 2,51 & & 3,98 & 3 . & & 3,85 & 3,63 & & $4.193,16$ \\
\hline $\begin{array}{l}\text { Respensa- } \\
\text { bilidad }\end{array}$ & 3,95 & 2,61 & Respeto & 3,95 & 3,12 & $\begin{array}{l}\text { Responsa- } \\
\text { bilidad }\end{array}$ & 3,62 & 2,24 & $\begin{array}{l}\text { Responsa: } \\
\text { billidad }\end{array}$ & 3,53 & 2,17 & $\begin{array}{l}\text { Responsa- } \\
\text { bilidad }\end{array}$ & $3,49 \quad 2,19$ \\
\hline
\end{tabular}

\section{CUADRO N" 3}

COMPARACIÓN DE LA AUTOESTIMA POR ESTUDIANTES Y NO ESTUDIANTES A TRAVES DE LA PRUEBA "t" DE STUDENT PARA GRUPOS INDEPENDIENTES

\begin{tabular}{|c|c|c|c|c|c|}
\hline \multirow[t]{2}{*}{ FACTORES } & \multicolumn{2}{|c|}{ ESTUDIANTES $(\mathrm{N}=2,785)$} & \multicolumn{2}{|c|}{ NO ESTUDIANTES $(\mathrm{N}=430)$} & \multirow[b]{2}{*}{ Hin } \\
\hline & M & $\mathrm{DE}$ & $\mathrm{M}$ & $\mathrm{DE}$ & \\
\hline $\mathrm{FI}$ & 13,33 & 2.81 & 9.41 & 6,51 & $21,36 \cdots$ \\
\hline $\mathrm{F} 2$ & 15.26 & 2,16 & 16,30 & 1.05 & $-9,80 \cdots$ \\
\hline F 3 & 3,00 & 1,23 & 3.67 & 1.06 & $-10.56 * * \bullet$ \\
\hline $\mathrm{F} 4$ & 1.76 & 1,04 & 1.93 & 0.90 & $-3,30 * \cdots$ \\
\hline AUTOESTIMA GENERAL & 33,34 & 5.04 & 31,30 & 7,30 & $7,31 \cdots$ \\
\hline
\end{tabular}

CUADRO N 4

COMPARACIÓN DE LA AUTOESTIMA POR LUGAR DE PROCEDENCIA A TRAVES DE. LA PRUEBA " $t$ " DE STUDENT PARA GRUPOS INDEPENDIENTES

\begin{tabular}{|c|c|c|c|c|c|}
\hline \multirow[t]{2}{*}{ FACTORES } & \multicolumn{2}{|c|}{ LIMA $\left(\mathrm{N}^{\circ} 2,548\right)$} & \multicolumn{2}{|c|}{ PROVINCIA (N* 667) } & \multirow[b]{2}{*}{ "to" } \\
\hline & M & DE & $\mathrm{M}$ & $\mathrm{DE}$ & \\
\hline $\mathrm{FI}$ & 13.13 & 3,35 & 11.55 & 4.92 & $9,71 * *$ \\
\hline $\mathrm{F} 2$ & 15.33 & 2,13 & 15,65 & 1.81 & $-3,61 * \cdots$ \\
\hline F 3 & 3,07 & 1.24 & 3.19 & 1.18 & $-2,42^{\circ}$ \\
\hline $\mathrm{F} 4$ & 1.78 & 1.04 & 1,77 & 0,97 & 0.16 \\
\hline AUTOESTIMA GENERAL & 33,30 & 5,28 & 32,18 & 5,91 & $4,78 * * a$ \\
\hline
\end{tabular}




\section{CUADRO N" 5}

COMPARACIÓN DE LA AUTOESTIMA POR GENERO A TRAVES DE LA PRUEBA "T" DE STUDENT PARA GRUPOS INDEPENDIENTES

\begin{tabular}{|c|c|c|c|c|c|}
\hline \multirow[t]{2}{*}{ FACTORES } & \multicolumn{2}{|c|}{ MASCULINO $(\mathrm{N}=1.632)$} & \multicolumn{2}{|c|}{ FEMENINO $(\mathrm{N}=1.583)$} & \multirow[b]{2}{*}{ "t" } \\
\hline & M & $\mathrm{DE}$ & $\mathrm{M}$ & DE & \\
\hline $\mathrm{Fl}$ & 12.59 & 3,88 & 13,02 & 3,67 & $-3.22 * 0$ \\
\hline $\mathrm{F2}$ & 15,31 & 2,12 & 15.48 & 2,02 & $-2,29^{*}$ \\
\hline F3 & 3,16 & 1,26 & 3.02 & 1.19 & $3,25 \cdots$ \\
\hline F4 & 1,71 & 1,03 & 1,85 & 1.01 & $-3,65 * \cdots$ \\
\hline AUTOESTIMA GENERAL & 32,78 & 5,57 & 33,37 & 5,28 & $-3,06 * *$ \\
\hline
\end{tabular}

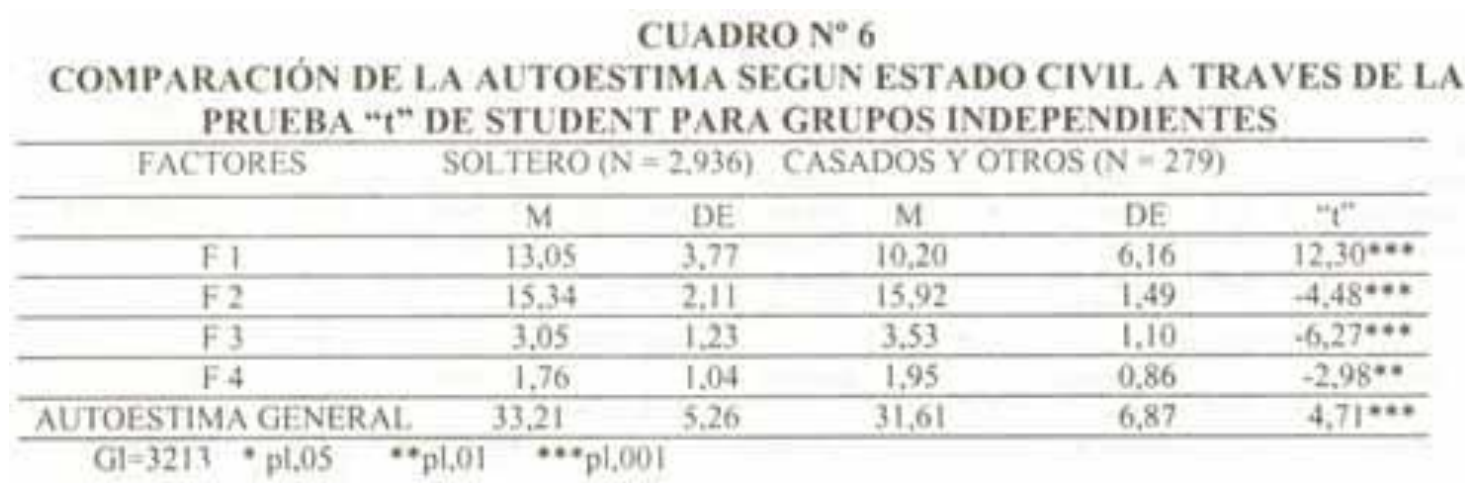

\section{CUADRO N"7}

COMPARACIÓN DE LA AUTOESTIMA SEGUN LA EDAD A TRAVES DEL ANÁLISIS DE VARIANZA DE UN FACTOR

\begin{tabular}{|c|c|c|c|c|c|}
\hline FACTORES & 18 a $20\left(N^{2} 1,59\right)$ & 21 a 30 (N"1,619) & 31 a $40\left(N^{2}-233\right)$ & 41 a más $\left(\mathrm{N}^{-103)}\right.$ & \\
\hline & $\mathrm{M}$ & $\mathrm{M}$ & $\mathrm{M}$ & $\bar{M}$ & $a F_{n}$ \\
\hline FI & 13,35abc & 12,95 ade & $10,69 \mathrm{bdf}$ & $8.60 \mathrm{cef}$ & 81.96 \\
\hline $\mathrm{F} 2$ & $15.26 \mathrm{ab}$ & $15,37 \mathrm{~cd}$ & $16,00 \mathrm{ac}$ & $16,04 \mathrm{bd}$ & 11.86 \\
\hline F 3 & $2.95 \mathrm{ab}$ & $3.08 \mathrm{~cd}$ & $3.70 \mathrm{ac}$ & $3,57 \mathrm{bd}$ & 30.01 \\
\hline $\mathrm{F} 4$ & $1.78 \mathrm{a}$ & $1.73 b$ & $1,99 \mathrm{ab}$ & 2,01 & 6,05 \\
\hline AUTOESTIMA GENERAL. & $33,34 a$ & $33.14 \mathrm{~b}$ & $32,36 \mathrm{c}$ & $30.22 \mathrm{abc}$ & 11,95 \\
\hline $\begin{array}{l}\text { GI } 1=3 \\
\text { GI } 2=3210\end{array}$ & $\cdots$ pl,001 & & & & \\
\hline
\end{tabular}

\section{DISCUSIÓN}

La autoestima como los valores surgen en el hombre y los acompañan a lo largo de su existencia, permitiéndole actuar e interactuar con personas, grupos u organizaciones. De manera que los resultados de la presente investigación estaría confirmando la relación entre la autoestima y los valores organizacionales analizados en estudiantes universitarios y no estudiantes universitarios.

Robbinson, (1994) consideraba que toda persona posee una jerarquía de valores que constituye su sistema de valores y este " ... sistema se identifica en razón de la importancia que se conceda a los valores ... ". Este sistema de valores es el que trasmiten los fundadores 
y directivos a sus organizaciones $\mathrm{y}$, de modo similar, son trasmitidos por los empleados que se van incorporando a ella, llegando a un proceso de influencia mutua para luego constituir el sistema de valores de la organización.

En 1998, Loli y colaboradores analizan una muestra de 105 gerentes y encuentran que la puntualidad era el valor más importante. En 1999, analizan en 1464 estudiantes universitarios quedando este valor desplazado al cuarto lugar en importancia, en cambio le dan prioridad a la limpieza, el servicio al cliente y la calidad del producto o servicio; es más, con este objeto se sacrifica la honradez, la responsabilidad y el respeto a la persona que son ubicados en los últimos lugares en importancia.

Ciertamente, es innegable que la percepción organizacional esté influida por el sistema individual de valores pero es interesante ver la variación de dicha jerarquía de una muestra a otra. Tal vez, como dicen, se debe a la “... experiencia de los gerentes por mejorar la disciplina, por demostrar eficacia frente a sus clientes, por cuidar su imagen, etc., los lleva a jerarquizar sus valores organizacionales de modo distinto, valores que aún no se perciben mientras no se tiene experiencia laboral o se es estudiante, o es que estamos frente a un futuro próximo diferente, acaso voraz y despiadado, como exige el sistema económico actual? .. " (Loli y col., 1999); más todavía si se toma en cuenta que los. valores tienen un gran impacto en el desempeño de las organizaciones (Korrer y Heskett, en Stoner y otros, 1996), o es que para los jóvenes universitarios los valores "... representan la convicción básica de que una forma de conducta específica o de condición última de la vida son preferibles, en términos personales o sociales a otra forma de conducta contrarias u opuestas ... " como afirma Rokeach (1973).

Esta vez, con una muestra ampliada ( 3,215 sujetos) tanto en estudiantes como en no estudiantes universitarios, los investigadores encuentran que el valor más importante es el servicio al cliente, seguido de limpieza y calidad del producto y del servicio y los menos importantes son la honradez, respeto a la persona y la responsabilidad, similar a lo encontrado por los mismos autores en una muestra menor en 1999. Separada la muestra, los estudiantes universitarios arrojan los mismos resultados, mientras que los no estudiantes le dan mayor importancia al servicio al cliente, limpieza y confianza y menor importancia a la honradez, la puntualidad y la responsabilidad. Esto puede ser demostrativo de que la madurez y la experiencia laboral permiten reordenar el sistema de valores en las personas y en las organizaciones, dándole prioridad a la confianza y sacrificando la puntualidad, antes que al valor respeto a la persona. De hecho, esto está relacionado también con la cultura organizacional y familiar, por cuanto el estudio sólo de los 105 gerentes (1998) le daba especial importancia a la puntualidad como valor, aunque no era necesariamente practicado.

Tratándose de los valores y de la autoestima, las personas de autoestima muy baja le dan más importancia al servicio al cliente, limpieza y calidad del producto o servicio y menos importancia a la honradez, respeto a la persona y a la responsabilidad. Postura que se mantiene en los que tienen la autoestima baja e intermedia, salvo el valor respeto a la persona que se ubica en el último lugar en los que tienen la autoestima baja y la puntualidad que se eleva al cuarto lugar, y se mantiene en esta posición hasta en aquellos que tienen autoestima alta. Sin embargo, en los que tienen la auto estima alta y muy alta, el valor limpieza tiene mas importancia que ningún otro, tal vez porque una elevación de la autoestima muy alta linda con los intereses personales y el narcisismo. 
Parece innegable que el "... punto de partida en la inductiva de los valores (positivos)... sea el incremento de la autoestima ... " (Mora, 1996), pero el exceso en el incremento de la autoestima parece distorsionar la consonancia con los valores (Loli, 1997), y no como se afirma que, la autoestima es el resultado que tiene el individuo de la "internalización de las normas y los valores del grupo social y de su adecuado desempeño" (Sherif y Sherif, 1996); por cuanto la interacción social como el desempeño son sólo el resultado de la relación consonante entre la autoestima y los valores.

Independientemente a la discusión anterior, lo cierto es que la autoestima " ... juega un papel modulador en la relación entre el clima organizacional y la satisfacción con el trabajo" (De la Poza y Prior, 1988; Mossholder, 1981; Adler, 1980), de manera que " ... el conflicto de función tiene relación negativa con la satisfacción laboral, el desempeño, el compromiso organizacional y la participación que da autoestima ... "; es decir, cuanto mayor es el conflicto de función menor será la participación que da autoestima. Lo cierto es que la autoestima reforzada es capaz de elevar la productividad e ir mucho más allá para mejorar su actitud en el servicio, elevando además su autoestima y el cambio personal hasta sentirse orgulloso, satisfecho con su empleo. "Este cambio de percepción laboral sólo es posible, como establece Yerkes (1978), con el desarrollo de la autoestima hasta el nivel que Maslow (1952) señala como nivel de autorrealización personal, que nosotros llamamos nivel de servicio" (Cruz, 1997), más allá del cual puede ser peligroso.

Loli y col. (1999), halló que existe correlación estadística significativa entre la autoestima y los valores organizacionales, siendo positiva en limpieza, y negativa en responsabilidad y respeto a la persona; en otros términos, cuanto mayor era la autoestima mayor era el valor que se le otorgaba a la limpieza, y menor la valoración a la responsabilidad y el respeto a la persona. Parece que estamos frente a una situación innegable en que el exceso de autoestima personal conduce a presumir y desalentar su responsabilidad organizacional y también el respeto a las personas. Entonces, no cabe hablar sólo de autoestima positiva o negativa, alta o baja sino que existe un máximo más allá del cual la autoestima es perjudicial, situación en la cual, la conducta personal como organizacional son anómalas, donde sólo la rectitud de valores sólidos sería capaz de controlar.

Nuestros resultados nos dicen también que mientras los estudiantes universitarios afirman su autoestima en el conocimiento de si mismo; los egresados, graduados y postgraduados (no estudiantes universitaríos) basan su autoestima en la realización personal, el respeto y la confianza que les permita crecer, como decía Maslow (1952) en la autorrealización. Aunque otros autores, como Cruz (1997), afirman que " ... cuando los valores guían la autoestima, se alcanza el equilibrio ético ... ", nosotros seguimos creyendo que los valores se construyen sobre la base de la autoestima, aunque no necesariamente es guía de los valores, porque éstos pueden distorsionarse si no hay una autoestima sólida y ésta es muy alta.

Ahora bien, el análisis comparativo de la autoestima con algunos rasgos demográficos (procedencia, genero, estado civil edad) nos muestra que existen diferencias significativas en todos los casos, siendo más alta la autoestima (la puntuación promedio) en los que proceden de Lima que aquellos que proceden de Provincia; en las mujeres más que en los hombres; en los solteros más que en los casados, viudos y convivientes; en los sujetos de 40 años y menores más que en 'los de 41 años y mayores. 
En el análisis Factor por Factor, se encuentran diferencias similares, salvo cuando se compara la muestra por procedencia donde en el Factor Autoconfianza (como componente de la autoestima) no arroja diferencia significativa, indicándonos que los de Provincia como los de Lima obtienen puntaje equivalente. Los hombres tienen el puntaje promedio más alto sólo en el Factor autorrespeto. Los solteros tienen el puntaje promedio más alto sólo en el Factor autoconocimiento. Y, respecto a la edad, el puntaje promedio en el Factor autoconocimiento disminuyen a medida que aumenta la edad y a la inversa, aumenta en autorrealización, autorespeto y Autoconfianza.

Los resultados revisados son coherentes con lo encontrado por los mismos autores en 1999, donde se halla una autoestima baja en varones y autoestima media en mujeres, permitiéndonos inferir que la influencia familiar más que la cultural es dominante en el desarrollo de la autoestima, persistiendo incluso en esta etapa de la formación profesional. Por consiguiente, parece indiscutible reforzar la autoestima tanto en varones como en mujeres con el objeto de elevar la productividad e ir mucho más allá para mejorar su actitud en el servicio (Cruz, 1997), sin menoscabar el respeto a la persona que parece ser el valor más afectado a la voracidad del sistema económico actual.

\section{CONCLUSIONES}

Las conclusiones a las que se puede arribar a partir del presente estudio son:

1. Los estudiantes universitarios le dan más importancia en su sistema de valores organizacionales al servicio al cliente, la limpieza y la calidad del producto o servicio y sacrifican la honradez, el respeto a la persona y la responsabilidad.

2. Los estudiantes no universitarios (egresados, graduados, posgraduados) prefieren, en su sistema de valores organizacionales al servicio al cliente, la limpieza y la confianza y sacrifican la honradez, la puntualidad y la responsabilidad.

3. Las personas de autoestima muy baja le dan más importancia al servicio al cliente, la limpieza y la calidad del producto o servicio y menos importancia a la honradez, el respeto a la persona y a la responsabilidad. Esta misma postura caracteriza a aquellos que tienen autoestima baja e intermedia. Sin embargo, hay dos valores que se movilizan: el valor respeto a la persona que se desplaza hasta el último lugar en aquellos que tienen autoestima baja y el valor puntualidad que se eleva al cuarto lugar en quienes tienen el nivel de autoestima intermedia y manteniéndose hasta aquellos que tienen la autoestima alta. En las personas que tienen autoestima alta y muy alta predomina el valor limpieza y mejora el valor disciplina. Los demás valores se mantienen inalterables.

4. La autoestima de los estudiantes universitarios alcanzan puntuaciones promedio más elevadas que la autoestima de los estudiantes no universitarios.

5. La autoestima de los estudiantes universitarios y no estudiantes universitarios que proceden de Lima alcanzan puntuaciones promedio más elevadas que aquellos que proceden de Provincia. 
6. La autoestima de los estudiantes universitarios y no estudiantes universitarios hombres alcanzan puntuaciones promedio más bajas que las mujeres.

7. La autoestima de los estudiantes universitarios y no estudiantes universitarios solteros alcanzan puntuaciones promedio más elevadas que los casados, viudos y convivientes.

8. La autoestima de los estudiantes universitarios y no estudiantes universitarios de 41 años de edad a más edad alcanzan puntuaciones promedio menores que aquellos participantes entre 18 a 40 años de edad. Es decir, los puntajes promedio disminuyen a medida que aumenta la edad, probablemente por que han cubierto sus expectativas y logrado estabilidad y por que su autoestima se basa fundamentalmente en la autorrealización, el autorrespeto y la autoconfianza. 


\section{REFERENCIAS}

ASPE, Virginia; LOPEZ, Ana. Hacia un desarrollo humano: Valores, actitudes y hábitos. México, Edit. Limusa, 1999.

BONET, José. Autoestima. España, Edit. Sal Terrae, 1994.

CLEMES, Harris y BEAN, Reynold. Cómo desarrollar la autoestima en los niños. España, Edit. Debate S.A., 1996.

CRUZ RAMIREZ, José. Autoestima y gestión de la calidad. México, Edit. Iberoamericana, 1997.

HUNTER, Lewis. La cuestión de los valores humanos. España, Barcelona, Edit. Gedisa, 1998.

LEBOW, Rob. La clave de la productividad empresarial. México, Panorama Editorial S.A.1995.

LOLI, Alejandro; LOPEZ, Ernestina. Autoestima y valores en la calidad y la excelencia. Lima, Perú, Inst. de Investigaciones Psicológicas, UNMSM, 1997.

LOLI, Alejandro; LOPEZ, Ernestina y cols.

La autoestima y los valores en las empresas de Lima. Perú, Inst. de Investigaciones Psicológicas, UNMSM, 1998.

LOLI, Alejandro; LOPEZ, Ernestina. Autoestima y valores en estudiantes de una universidad pública y privada de Lima. Perú, Inst. de Investigaciones Psicológicas, UNMSM,1999.

LOLI, Alejandro; LOPEZ, Ernestina. Inventario de autoestima para adultos (ALPEL, Forma-AD Lima, Perú, Inst. de Investigaciones Psicológicas, UNMSM, 2000.

MASLOW, Abraham. El hombre autorrealizado. Barcelona, Edi1. Kairós, 1979.

MEYERS, David G. Psicología social. México, Edi1. McGraw Hill, 1995.

MORA, Guillermo; SARMIENTO, Enma; OSSA, John. Valores humanos y actitudes positivas. Colombia, McGraw Hill, 1995.

O'CONNOR, Michael. Administración por valores. Colombia, Edit. Norma, 1997.

OÑATE, M.P. El Autoconcepto, formación, medida e implicaciones en la personalidad. Madrid, Edil. Narcea, 1989.

ROBBINS, Stephen. Comportamiento organizacional. México, Edit. Prentice Hall Hispanoamericana, 1994.

RODRIGUEZ, Mauro. Los valores, clave de la excelencia. México, Edit. McGraw Hil, 1993.

STONER, James; FREEMAN, Edward; GUILBET, Daniel. Administración. México, Edit. Prentice Hall Hispanoamericana, 1996.

THEVENET, Maurice. Auditoría de la cultura empresarial. España, Madrid, Edit. Díaz de Santos, 1992. 